\title{
CORRECTION
}

View Article Online

View Journal I View Issue

W) Check for updates

Cite this: RSC Adv., 2021, 11, 38690

DOI: $10.1039 / \mathrm{d} 1 \mathrm{ra90173g}$

rsc.li/rsc-advances

\section{Correction: The role of substituted pyridine Schiff bases as ancillary ligands in the optical properties of a new series of fac-rhenium(I) tricarbonyl complexes: a theoretical view}

\author{
Rosaly Morales-Guevara, ${ }^{\text {ab }}$ Juan A. Fuentes, ${ }^{c}$ Dayán Paez-Hernández ${ }^{\star a b}$ \\ and Alexander Carreño*ab \\ Correction for 'The role of substituted pyridine Schiff bases as ancillary ligands in the optical properties of \\ a new series of fac-rhenium(I) tricarbonyl complexes: a theoretical view' by Rosaly Morales-Guevara et al., \\ RSC Adv., 2021, 11, 37181-37193, DOI: 10.1039/D1RA05737E.
}

The authors regret that the email address of one of the corresponding authors (Dayán Paez-Hernández) was shown incorrectly in the original manuscript. The corrected email is as follows: dayan.paez@unab.cl.

The Royal Society of Chemistry apologises for these errors and any consequent inconvenience to authors and readers.

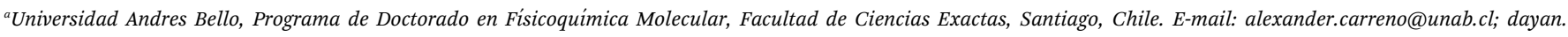
paez@unab.cl

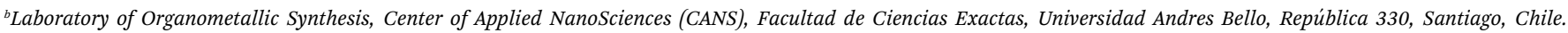
E-mail: alexander.carreno@unab.cl; dayan.paez@unab.cl

${ }^{c}$ Laboratorio de Genética y Patogénesis Bacteriana, Facultad de Ciencias de la Vida, Universidad Andres Bello, República 330, Santiago, Chile 